\title{
Liter per Day per Kilogram per Meter Squared
}

National Cancer Institute

\section{Source}

National Cancer Institute. Liter per Day per Kilogram per Meter Squared. NCI Thesaurus.

Code C120727.

A unit of concentration equal to liter per day divided by kilog ram per meter squared. 